\title{
Hybrid Rational Haar Wavelet and Block Pulse Functions Method for Solving Population Growth Model and Abel Integral Equations
}

\author{
E. Fathizadeh, R. Ezzati, and K. Maleknejad \\ Department of Mathematics, Islamic Azad University, Karaj Branch, Karaj, Iran \\ Correspondence should be addressed to R. Ezzati; ezati@kiau.ac.ir
}

Received 12 August 2016; Revised 14 November 2016; Accepted 1 December 2016; Published 17 January 2017

Academic Editor: Michael Vynnycky

Copyright (C) 2017 E. Fathizadeh et al. This is an open access article distributed under the Creative Commons Attribution License, which permits unrestricted use, distribution, and reproduction in any medium, provided the original work is properly cited.

We use a computational method based on rational Haar wavelet for solving nonlinear fractional integro-differential equations. To this end, we apply the operational matrix of fractional integration for rational Haar wavelet. Also, to show the efficiency of the proposed method, we solve particularly population growth model and Abel integral equations and compare the numerical results with the exact solutions.

\section{Introduction}

Fractional calculus is a field of applied mathematics that deals with derivatives and integrals of arbitrary orders (including complex orders). It is also known as generalized integral and differential calculus of arbitrary order. Fractional differential equations are generalized from classical integer-order ones, which are obtained by replacing integer-order derivatives by fractional ones. In recent years, fractional calculus and differential equations have found enormous applications in mathematics, physics, chemistry, and engineering [1-4]. A large class of dynamical systems appearing throughout the field of engineering and applied mathematics is described by fractional differential equations. For that reason, reliable and efficient techniques for the solution of fractional differential equations are indeed required. The most frequently used methods are Walsh functions [5], Laguerre polynomials [6], Fourier series [7], Laplace transform method [8], the Haar wavelets [9], Legendre wavelets [10-12], and the Chebyshev wavelets $[13,14]$. Kronecker operational matrices have been developed by Kilicman and $\mathrm{Al}$ Zhour for some applications of fractional calculus [15]. Recently, in [16], the authors proposed a new method based on operational matrices to solve fractional Volterra integral equations.

Recently, many authors applied operational matrices of integration and derivative to reduce the original problem into an algebraic one. According to this fact that the orthogonal polynomials play an important role to solve integral and differential equations, many researchers constructed operational matrix of fractional and integer derivatives for some types of these polynomials, such as Flatlet oblique multiwavelets [17, 18], B-spline cardinal functions [19], Legendre polynomials, Chebyshev polynomials, and CAS wavelets [20]. The main aim of this paper is to use an operational matrix of fractional integration to reduce a nonlinear fractional integro-differential equation to nonlinear algebraic equations.

The rest of the paper is organized as follows: In Section 2, we introduce some basic mathematical preliminaries that we need to construct our method. Also, we recall the basic definitions from block pulse functions and fractional calculus. In Section 3, we recall definition of rational Haar wavelet. In Section 4, we apply proposed method to solve fractional population growth model and Abel integral equations. Section 5 is devoted to convergence and error analysis. Finally, in Section 6, conclusion of numerical results is presented.

\section{Preliminaries}

In this section, we recall some basic definitions from fractional calculus and some properties of integral calculus which we shall apply to formulate our approach. 
The Riemann-Liouville fractional integral operator $I^{\alpha}$ of order $\alpha \geq 0$ on the usual Lebesgue space $L^{1}[0, b]$ is given by [21]

$$
\left(I^{\alpha} u\right)(x)= \begin{cases}\frac{1}{\Gamma(\alpha)} \int_{0}^{x}(x-s)^{\alpha-1} u(s) d s & \alpha>0, \\ u(x) & \alpha=0 .\end{cases}
$$

The Riemann-Liouville fractional derivative of order $\alpha>0$ is normally defined as

$$
D^{\alpha} u(x)=\left(\frac{d}{d x}\right)^{m} I^{m-\alpha} u(x), \quad(m-1<\alpha \leq m),
$$

where $m$ is an integer number.

The fractional derivative of order $\alpha>0$ in the Caputo sense is given by [21]

$$
\begin{array}{r}
D_{*}^{\alpha} u(x)=\frac{1}{\Gamma(m-\alpha)} \int_{0}^{x}(x-s)^{m-\alpha-1} u^{(m)}(s) d s, \\
\quad(m-1<\alpha \leq m),
\end{array}
$$

where $m$ is an integer, $\alpha>0$, and $u^{(m)} \in L^{1}[0, b]$.

The useful relation between the Riemann-Liouville operator and Caputo operator is given by the following expression:

$$
\begin{array}{r}
I^{\alpha} D_{*}^{\alpha} u(x)=u(x)-\sum_{k=0}^{m-1} u^{(k)}\left(0^{+}\right) \frac{x^{k}}{k !}, \\
\quad(m-1<\alpha \leq m),
\end{array}
$$

where $m$ is an integer, $\alpha>0$, and $u^{(k)} \in L^{1}[0, b]$.

An $m$-set of block pulse functions (BPFs) in the region of $[0, T)$ is defined as follows:

$$
b_{i}(t)= \begin{cases}1, & i h \leq t<(i+1) h, \\ 0, & \text { O.W, }\end{cases}
$$

where $i=1,2, \ldots, m-1$ with positive integer values for $m$ and $h=T / m$. There are some properties for BPFs, for example, disjointness, orthogonality, and completeness.

The set of BPFs may be written as an $m$-vector as

$$
B(t)=\left[b_{0}(t), \ldots, b_{m-1}(t)\right]^{T},
$$

where $t \in[0,1)$.

A function $f(t) \in L^{2}([0,1))$ may be expanded by the BPFs as

$$
f(t) \simeq \sum_{i=0}^{m-1} f_{i} b_{i}(t)=F^{T} B(t)=B^{T}(t) F,
$$

where $B(t)$ is given by (6) and $F$ is an $m$-vector given by

$$
F=\left[f_{0}, \ldots, f_{m-1}\right]^{T},
$$

and the block pulse coefficients $f_{i}$ are obtained as

$$
f_{i}=\frac{1}{h} \int_{i h}^{(i+1) h} f(t) d t .
$$

The integration of vector $B(t)$ defined in (6) may be obtained as

$$
\int_{0}^{t} B(\tau) d \tau \simeq \Upsilon B(t),
$$

where $\Upsilon$ is called operational matrix of integration which can be represented by

$$
\Upsilon=\frac{h}{2}\left(\begin{array}{ccccc}
1 & 2 & 2 & \cdots & 2 \\
0 & 1 & 2 & \cdots & 2 \\
\vdots & \vdots & \vdots & \ddots & \vdots \\
0 & 0 & 0 & \cdots & 1
\end{array}\right) .
$$

Kilicman and Al Zhour (see [15]) have given the block pulse operational matrix of fractional integration $F^{\alpha}$ as follows:

$$
\left(I^{\alpha} B_{m}\right)(t) \simeq F^{\alpha} B_{m}(t),
$$

where

$$
F^{\alpha}=\frac{1}{m} \frac{1}{\Gamma(\alpha+2)}\left(\begin{array}{cccccc}
1 & \xi_{1} & \xi_{2} & \xi_{3} & \cdots & \xi_{m-1} \\
0 & 1 & \xi_{1} & \xi_{2} & \cdots & \xi_{m-2} \\
0 & 0 & 1 & \xi_{1} & \cdots & \xi_{m-3} \\
\vdots & \vdots & \ddots & \ddots & & \vdots \\
0 & 0 & \cdots & 0 & 1 & \xi_{1} \\
0 & 0 & 0 & \cdots & 0 & 1
\end{array}\right),
$$

and $\xi_{k}=(k+1)^{\alpha+1}-2 k^{\alpha+1}+(k-1)^{\alpha+1}$.

\section{Rational Haar Wavelets}

The Haar functions are an orthogonal family of switched rectangular waveforms where amplitudes can differ from one function to another. The orthogonal set of Haar wavelet functions is defined in $[0,1)$ as follows (see [22]):

$$
h_{i}(t)=\frac{1}{\sqrt{m}} \begin{cases}2^{j / 2}, & \frac{k-1}{2^{j}} \leq t<\frac{k-1 / 2}{2^{j}}, \\ -2^{j / 2}, & \frac{k-1 / 2}{2^{j}} \leq t<\frac{k}{2^{j}}, \\ 0, & \text { O.W, }\end{cases}
$$

where $i=0,1,2, \ldots, m-1, m=2^{M+1}$ and $M$ is a positive integer, and $j$ and $k$ represent the integer decomposition of the index $i$, that is, $i=2^{j}+k-1, j=0,1, \ldots, M$, $k=1,2, \ldots, 2^{j}$. Also we have $h_{0}(t)=1 / \sqrt{m}$. This set of functions is complete, since any function $f \in L^{2}([0,1))$ can be expanded into Haar wavelets by

$$
f(t) \simeq \sum_{i=0}^{m-1} f_{i} h_{i}(t)=F^{T} \Psi(t),
$$

where

$$
\begin{aligned}
F & =\left[f_{0}, f_{1}, f_{2}, \ldots, f_{m-1}\right]^{T}, \\
\Psi(t) & =\left[h_{0}(t), h_{1}(t), h_{2}(t), \ldots, h_{m-1}(t)\right]^{T} .
\end{aligned}
$$


Operational Matrix of Fractional Integration. Equation (7) implies that rational Haar wavelets can be also expanded into $m$-term BPFs as

$$
h_{i}(t) \simeq \sum_{j=0}^{m-1} h_{i j} b_{j}(t)
$$

for $i=1,2, \ldots, m-1$. Clearly we have

$$
\Psi(t)=H_{m \times m} B(t)
$$

where

$$
H_{m \times m}
$$

$$
=\left(\begin{array}{cccc}
h_{0}\left(t_{0}\right) & h_{0}\left(t_{1}\right) & \cdots & h_{0}\left(t_{m-1}\right) \\
h_{1}\left(t_{0}\right) & h_{1}\left(t_{1}\right) & \cdots & h_{1}\left(t_{m-1}\right) \\
\vdots & \vdots & \cdots & \vdots \\
h_{m-1}\left(t_{0}\right) & h_{m-1}\left(t_{1}\right) & \cdots & h_{m-1}\left(t_{m-1}\right)
\end{array}\right) \text {, }
$$

and $t_{i}=(i+1 / 2) / m$, for $i=0,1,2, \ldots, m-1$ (see $\left.[15,23]\right)$.

For example, for $M=2$, the Haar operational matrix into BPFs can be expressed as

$$
H_{8 \times 8}=\left(\begin{array}{cccccccc}
0.3536 & 0.3536 & 0.3536 & 0.3536 & 0.3536 & 0.3536 & 0.3536 & 0.3536 \\
0.3536 & 0.3536 & 0.3536 & 0.3536 & -0.3536 & -0.3536 & -0.3536 & -0.3536 \\
0.5000 & 0.5000 & -0.5000 & -0.5000 & 0 & 0 & 0 & 0 \\
0 & 0 & 0 & 0 & 0.5000 & 0.5000 & -0.5000 & -0.5000 \\
0.7071 & -0.7071 & 0 & 0 & 0 & 0 & 0 & 0 \\
0 & 0 & 0.7071 & -0.7071 & 0 & 0 & 0 & 0 \\
0 & 0 & 0 & 0 & 0.7071 & -0.7071 & 0 & 0 \\
0 & 0 & 0 & 0 & 0 & 0 & 0.7071 & -0.7071
\end{array}\right) .
$$

Let

$$
\left(I^{\alpha} \Psi\right)(t) \simeq P_{m \times m}^{\alpha} \Psi(t),
$$

where matrix $P_{m \times m}^{\alpha}$ is called the Haar wavelet operational matrix of fractional integration. Using (18) and (12), we have

$$
\begin{aligned}
\left(I^{\alpha} \Psi\right)(t) & \simeq\left(I^{\alpha} H_{m \times m} B_{m}\right)(t)=H_{m \times m}\left(I^{\alpha} B_{m}\right)(t) \\
& \simeq H_{m \times m} F^{\alpha} B_{m}(t) .
\end{aligned}
$$

By (21) and (22), we get

$$
P_{m \times m}^{\alpha} \Psi(t) \simeq H_{m \times m} F^{\alpha} B_{m}(t) ;
$$

therefore the Haar wavelet operational matrix of fractional integration $P_{m \times m}^{\alpha}$ is as follows:

$$
P_{m \times m}^{\alpha}=H_{m \times m} F^{\alpha} H_{m \times m}^{T} .
$$

For example, with $M=2$, the Haar operational matrix into BPFs can be expressed as

$$
P_{8 \times 8}^{1 / 2}=\left(\begin{array}{cccccccc}
0.0940 & 0.1719 & 0.2226 & 0.2637 & 0.2991 & 0.3307 & 0.3595 & 0.3862 \\
0.0940 & 0.1719 & 0.2226 & 0.2637 & 0.1110 & -0.0132 & -0.0858 & -0.1411 \\
0.1330 & 0.2431 & 0.0489 & -0.1134 & -0.0738 & -0.0349 & -0.0226 & -0.0162 \\
0 & 0 & 0 & 0 & 0.1330 & 0.2431 & 0.0489 & -0.1134 \\
0.1881 & -0.0323 & -0.0544 & -0.0194 & -0.0112 & -0.0076 & -0.0056 & -0.0043 \\
0 & 0 & 0.1881 & -0.0323 & -0.0544 & -0.0194 & -0.0112 & -0.0076 \\
0 & 0 & 0 & 0 & 0.1881 & -0.0323 & -0.0544 & -0.0194 \\
0 & 0 & 0 & 0 & 0 & 0 & 0.1881 & -0.0323
\end{array}\right) .
$$


TABLE 1: Comparison of exact value of $u_{\max }$ with the proposed method (RHM), ADM and HPM.

\begin{tabular}{|c|c|c|c|c|c|c|}
\hline \multirow[b]{2}{*}{$\kappa$} & \multicolumn{4}{|c|}{$\alpha=1$} & \multicolumn{2}{|c|}{$\alpha=3 / 4$} \\
\hline & Exact. $u_{\max }$ & RHM & $\mathrm{ADM}$ & HPM & RHM & HPM \\
\hline 0.02 & 0.9234271700 & 0.9234473344 & 0.9234270 & 0.922942037 & 0.9186276936 & 2.92000000 \\
\hline 0.04 & 0.8737199832 & 0.8737197006 & 0.8612401 & 0.873725344 & 0.8590308516 & 2.01600000 \\
\hline 0.1 & 0.7697414491 & 0.7697404269 & 0.7651130 & 0.765113089 & 0.7517051345 & 1.23200000 \\
\hline 0.2 & 0.6590503816 & 0.6590500286 & 0.6579123 & 0.659050432 & 0.6362736501 & 0.81130000 \\
\hline 0.5 & 0.4851902914 & 0.4851895440 & 0.4852823 & 0.485190290 & 0.4475550726 & 0.44320000 \\
\hline
\end{tabular}

\section{Implementation of the Method}

In this section, we present a computational method for solving the nondimensional fractional population growth model and Abel integral equations.

4.1. Population Growth Model. The Volterra model for nondimensional fractional population growth model is as follows:

$$
\begin{aligned}
& \kappa D_{*}^{\alpha} u(t)-u(t)+u^{2}(t)+u(t) \int_{0}^{t} u(\tau) d \tau=0, \\
& u(0)=u_{0}, 0<\alpha \leq 1 .
\end{aligned}
$$

The analytical solution (26) for $\alpha=1$ is (see [24])

$$
u(t)=u_{0} \exp \left(\frac{1}{\kappa} \int_{0}^{t}\left(1-u(\tau)-\int_{0}^{\tau} u(s) d s\right) d \tau\right) .
$$

The exact values of $u_{\max }$ were evaluated by using

$$
u_{\max }=1+\kappa \ln \left(\frac{\kappa}{1+\kappa-u_{0}}\right) \text {. }
$$

For solving (26), we first approximate $D_{*}^{\alpha} u(t)$ as

$$
D_{*}^{\alpha} u(t) \simeq U^{T} \Psi(t),
$$

where $U$ is an unknown vector which should be found and $\Psi(t)$ is the vector which is defined in (16). By using initial condition, $u(0)=u_{0}$, and (4), we have

$$
u(t) \simeq U^{T} P^{\alpha} \Psi(t)+u_{0} .
$$

By using (18) and (30), we conclude that

$$
u(t) \simeq U^{T} P^{\alpha} H_{m^{\prime} \times m^{\prime}} B_{m^{\prime}}(t)+u_{0}[1,1, \ldots, 1] B_{m^{\prime}}(t) .
$$

Let

$$
\begin{aligned}
A^{T} & =\left[a_{1}, a_{2}, \ldots, a_{m^{\prime}}\right] \\
& =U^{T} P^{\alpha} H_{m^{\prime} \times m^{\prime}}+u_{0}[1,1, \ldots, 1] .
\end{aligned}
$$

By using (31) and (32), we have $u(t) \simeq A^{T} B_{m^{\prime}}(t)$. From (5) we have

$$
u^{2}(t) \simeq\left[a_{1}^{2}, a_{2}^{2}, \ldots, a_{m^{\prime}}^{2}\right] B_{m^{\prime}}(t)=\widetilde{A}^{T} B_{m^{\prime}}(t) .
$$

Also, from (10) we have

$$
\begin{aligned}
\int_{0}^{t} u(\tau) d \tau & \simeq \int_{0}^{t} A^{T} B_{m^{\prime}}(\tau) d \tau \simeq A^{T} \Upsilon B_{m^{\prime}}(t) \\
& =C^{T} B_{m^{\prime}}(t),
\end{aligned}
$$

where $C^{T}=A^{T} \Upsilon$. By using (5), (31), and (34), we have

$$
u(t) \int_{0}^{t} u(\tau) d \tau \simeq \widetilde{U}^{T} B_{m^{\prime}}(t),
$$

where

$$
\widetilde{U}^{T}=\left[a_{1} c_{1}, a_{2} c_{2}, \ldots, a_{m^{\prime}} c_{m^{\prime}}\right] .
$$

Now by substituting (29), (31), (33), and (35) into (26), we obtain

$$
\left(\kappa U^{T} H_{m^{\prime} \times m^{\prime}}-A^{T}+\widetilde{A}^{T}+\widetilde{U}^{T}\right) B_{m^{\prime}}(t) \simeq 0 .
$$

By replacing $\simeq$ by $=$, we obtain the following system of nonlinear algebraic equations:

$$
\kappa U^{T} H_{m^{\prime} \times m^{\prime}}-A^{T}+\widetilde{A}^{T}+\widetilde{U}^{T}=0 .
$$

Finally, by solving this system, we obtain the approximate solution of the problem as $u(t) \simeq A^{T} B_{m^{\prime}}(t)$. As a numerical example, we consider the nonlinear fractional integrodifferential equation (26) with the initial condition $u(0)=$ 0.1 ; for more details, see Table 1 and Figures 1 and 2.

4.2. Abel Equations. Consider the generalized linear Abel integral equations of the first and second kinds, respectively, as [25]

$$
\begin{aligned}
& f(x)=\int_{0}^{x} \frac{y(t)}{(x-t)^{1-\alpha}} d t, \quad 0<\alpha<1, \\
& y(x)=f(x)+\int_{0}^{x} \frac{y(t)}{(x-t)^{1-\alpha}} d t, \quad 0<\alpha<1,
\end{aligned}
$$

where $f(x)$ and $y(x)$ are differentiable functions. Here, we apply fractional integration operational matrix of rational Haar wavelet to solve Abel integral equations as fractional integral equations.

Let $y(x)=U^{T} \Psi(x)$. Clearly, we can write (1) as follows:

$$
\int_{0}^{x} \frac{y(t)}{(x-t)^{1-\alpha}} d t=\Gamma(\alpha) I^{\alpha} y(x) .
$$




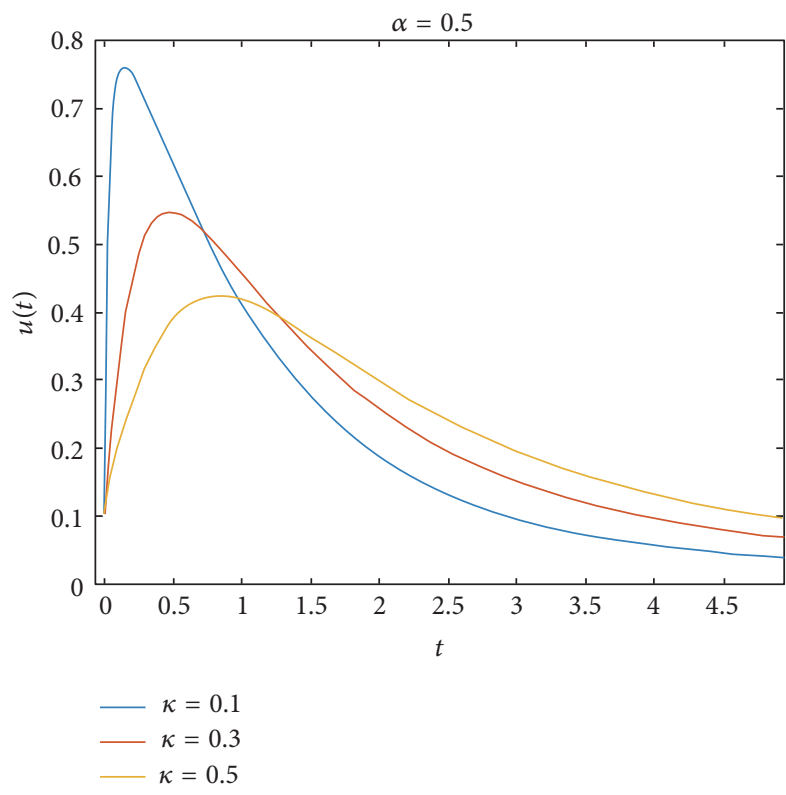

FIGURE 1: Numerical solutions of the fractional population growth model for different values of $\kappa$.

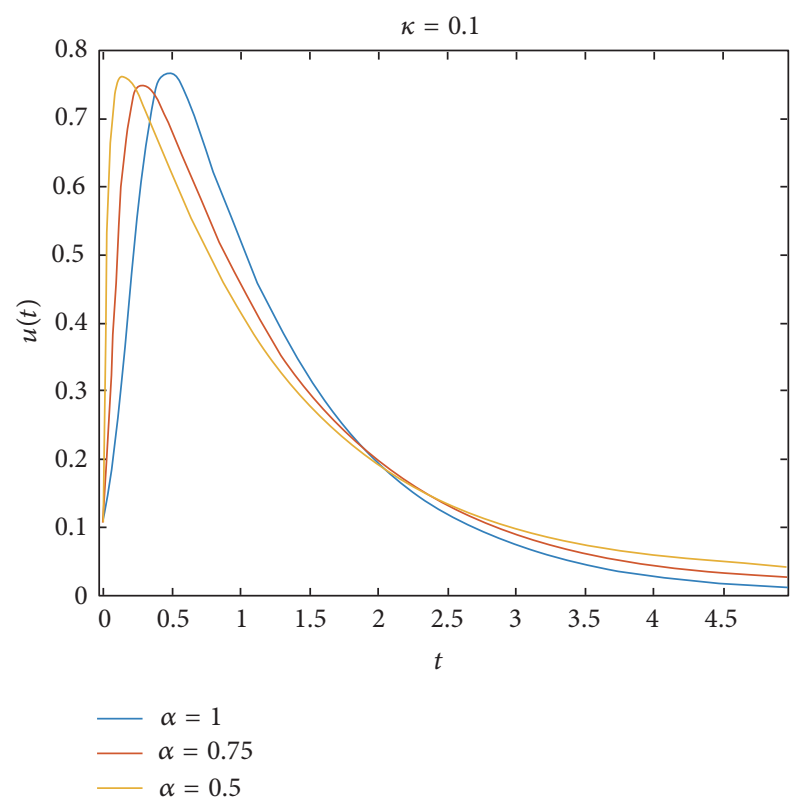

FIGURE 2: Numerical solutions of the fractional population growth model with $\kappa=0.1$ for different values of $\alpha$.

Now, by using (40), we obtain fractional form of Abel integral equations of the first and second kind, respectively, as the following form:

$$
\begin{aligned}
& f(x)=\Gamma(\alpha) U^{T} P^{\alpha} \Psi(x), \\
& f(x)=\left(U^{T}-\Gamma(\alpha) U^{T} P^{\alpha}\right) \Psi(x) .
\end{aligned}
$$

By collocating (41) in $t_{i}=(i+1 / 2) / m$, for $i=0,1,2, \ldots$, $m-1$, we obtain the following systems of algebraic equations:

$$
\begin{aligned}
& f\left(x_{i}\right)=\Gamma(\alpha) U^{T} P^{\alpha} \Psi\left(x_{i}\right), \\
& f\left(x_{i}\right)=\left(U^{T}-\Gamma(\alpha) U^{T} P^{\alpha}\right) \Psi\left(x_{i}\right) .
\end{aligned}
$$

Finally, by solving this system and determining $U$, we obtain the approximate solution of (41) as $y(x)=U^{T} \Psi(x)$.

Example 1. Consider the second kind Abel integral equation of the form

$$
y(x)=x^{2}+\frac{16}{15} x^{5 / 2}-\int_{0}^{x} \frac{y(t)}{(x-t)^{1 / 2}} d t .
$$

The exact solution is $y(x)=x^{2}$. $m^{\prime}$.

$$
\text { Let } y(x)=U^{T} \Psi(x) \text { and } X=(l-.5) / m^{\prime}, \text { for } l=1,2, \ldots,
$$

From (1), (18), and (21), we have

$$
\begin{aligned}
\int_{0}^{x} \frac{y(t)}{(x-t)^{1 / 2}} d t & =\Gamma\left(\frac{1}{2}\right) I^{\alpha}(y(x)) \\
& =\Gamma\left(\frac{1}{2}\right) I^{\alpha}\left(U^{T} \Psi(x)\right) \\
& =\Gamma\left(\frac{1}{2}\right) U^{T} P^{\alpha} H_{m^{\prime} \times m^{\prime}} B(x) .
\end{aligned}
$$

So, we get the algebraic equations as follows:

$$
\begin{gathered}
U^{T} H_{m^{\prime} \times m^{\prime}}-\left(X^{2}\right)^{T}-\frac{16}{15}\left(X^{5 / 2}\right)^{T} \\
+\Gamma\left(\frac{1}{2}\right) U^{T} P^{\alpha} H_{m^{\prime} \times m^{\prime}}=0 .
\end{gathered}
$$

By solving this system, we obtain the approximate solution of the problem as $y(x)=U^{T} \Psi(x)$. Figure 3 shows the plot of error for presented method and the exact solution of this example.

Example 2. Consider Abel integral equation of the first kind

$$
x=\int_{0}^{x} \frac{y(t)}{(x-t)^{4 / 5}} d t .
$$

The exact solution is $y(x)=(5 / 4)(\sin (\pi / 5) / \pi) x^{4 / 5}$.

To compare the numerical results and the exact solution, one can refer to Figure 4.

\section{Error Analysis}

In this section, we assume that $f(t)$ is a differentiable function and also $f^{\prime}(t)$ is bounded on the interval $[0,1]$; that is,

$$
\exists K>0 ; \quad \forall t \in[0,1]:\left|f^{\prime}(t)\right| \leqslant K .
$$

If $f_{m}(t)$ is the approximation of $f(t)$ as

$$
f(t) \simeq \sum_{i=0}^{m-1} f_{i} h_{i}(t),
$$




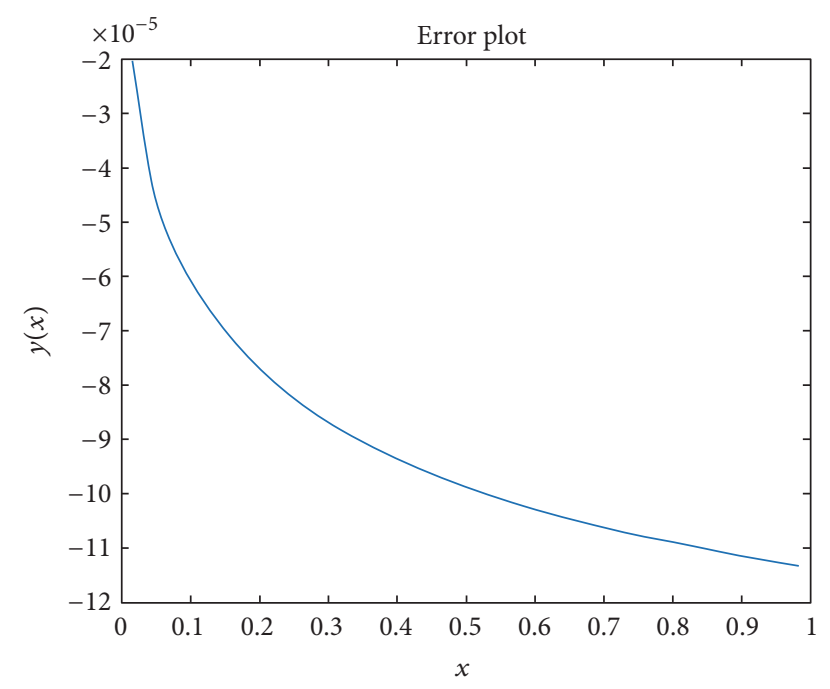

FIGURE 3: Error plot for Example 1 with $m^{\prime}=32$.

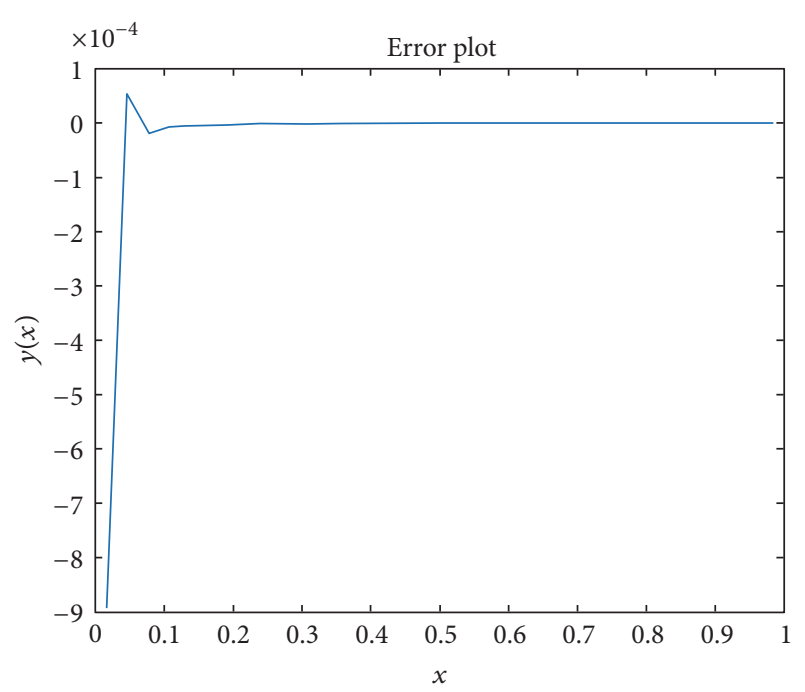

FIgURE 4: Error plot for Example 2 with $m^{\prime}=32$.

where $m=2^{M+1}$ and $M$ is a positive integer, the corresponding error function is denoted by $e_{m}(x)=f(x)-f_{m}(x)$.

Theorem 3. Suppose that $f(x) \in L^{2}[0,1]$ with bounded first derivative, $\left|f^{\prime}(t)\right| \leqslant K$, and $f(t) \simeq \sum_{i=0}^{m-1} f_{i} h_{i}(t)$. Then we have the error bound as follows:

$$
\left\|e_{m}(x)\right\|_{E}=\left\|f(x)-f_{m}(x)\right\|_{E} \leqslant \frac{K}{\sqrt{3} m},
$$

where $\left\|e_{m}(x)\right\|_{E}=\int_{0}^{1}\left(e_{m}(x)\right)^{2} d x$.

Proof. See [26].

In other words, by increasing $m$, the error function, $e_{m}(t)$, approaches to zero. If $e_{m}(t) \rightarrow 0$ when $m$ is sufficiently large enough, then the error decreases.

\section{Conclusion}

In this paper, we presented a numerical scheme for solving fractional population growth model and Abel integral equations of the first and second kinds. The method which is employed is based on the rational Haar wavelet. In Figures 1 and 2, the solutions of fractional population growth model for different values of $\kappa$ and $\alpha$ are shown. Table 1 represents the exact value of $u_{\max }$ and comparison of our used rational Haar method (RHM) with ADM (Adomian Decomposition Method [27]) and HPM (Homotopy-Padé Approximation Method [28]). By considering Abel integral equations of the first and second kinds as a fractional integral equation, we use fractional calculus properties for solving these singular integral equations. The fractional integration is described in the Riemann-Liouville sense. This matrix is used to approximate the numerical solution of the generalized Abel integral equations of the first and second kinds. Presented approach was based on the collocation method. Figures 3 and 4 show the plot of the error of presented method and the exact solution of Abel equations (Examples 1 and 2, resp.). The obtained results show that the used technique can be a suitable method to solve the fractional integro-differential equations.

\section{Competing Interests}

The authors declare that there is no conflict of interests regarding the publication of this paper.

\section{References}

[1] J. H. He, "Nonlinear oscillationwith fractional derivative and its applications," in Proceedings of the International Conference on Vibrating Engineering, vol. 98, pp. 288-291, Dalian, China, 1998.

[2] "Fractional calculus: some basic problems in continuum and statistical mechanics," in Fractals and Fractional Calculus in Continuum Mechanics, A. Carpinteri and F. Mainardi, Eds., vol. 378, pp. 291-348, Springer, New York, NY, USA, 1997.

[3] Y. A. Rossikhin and M. V. Shitikova, "Applications of fractional calculus to dynamic problems of linear and nonlinear hereditary mechanics of solids," Applied Mechanics Reviews, vol. 50, no. 1, pp. 15-67, 1997.

[4] R. T. Baillie, "Long memory processes and fractional integration in econometrics," Journal of Econometrics, vol. 73, no. 1, pp. 559, 1996.

[5] C.-F. Chen and C.-H. Hsiao, "Design of piecewise constant gains for optimal control via Walsh functions," IEEE Transactions on Automatic Control, vol. 20, no. 5, pp. 596-603, 1975.

[6] D.-H. Shih, F.-C. Kung, and C.-M. Chao, "Laguerre series approach to the analysis of a linear control system incorporating observers," International Journal of Control, vol. 43, no. 1, pp. 123-128, 1986.

[7] P. N. Paraskevopoulos, P. D. Sparis, and S. G. Mouroutsos, "The Fourier series operational matrix of integration," International Journal of Systems Science, vol. 16, no. 2, pp. 171-176, 1985.

[8] I. Podlubny, "The Laplace transform method for linear differential equations of the fractional order," Tech. Rep. UEF-02-94, Institute of Experimental Physics, Slovak Academy of Sciences, Kosice, Slovakia, 1994. 
[9] Y. Li and W. Zhao, "Haar wavelet operational matrix of fractional order integration and its applications in solving the fractional order differential equations," Applied Mathematics and Computation, vol. 216, no. 8, pp. 2276-2285, 2010.

[10] M. Ur Rehman and R. Ali Khan, "The Legendre wavelet method for solving fractional differential equations," Communications in Nonlinear Science and Numerical Simulation, vol. 16, no. 11, pp. 4163-4173, 2011.

[11] M. H. Heydari, M. R. Hooshmandasl, F. M. M. Ghaini, and F. Fereidouni, "Two-dimensional legendre wavelets for solving fractional poisson equation with dirichlet boundary conditions," Engineering Analysis with Boundary Elements, vol. 37, no. 11, pp. 1331-1338, 2013.

[12] M. H. Heydari, M. R. Hooshmandasl, C. Cattani, and M. Li, "Legendre wavelets method for solving fractional population growth model in a closed system," Mathematical Problems in Engineering, vol. 2013, Article ID 161030, 8 pages, 2013.

[13] Y. Li, "Solving a nonlinear fractional differential equation using Chebyshev wavelets," Communications in Nonlinear Science and Numerical Simulation, vol. 15, no. 9, pp. 2284-2292, 2010.

[14] Y. Wang and Q. Fan, "The second kind Chebyshev wavelet method for solving fractional differential equations," Applied Mathematics and Computation, vol. 218, no. 17, pp. 8592-8601, 2012.

[15] A. Kilicman and Z. A. A. Al Zhour, "Kronecker operational matrices for fractional calculus and some applications," Applied Mathematics and Computation, vol. 187, no. 1, pp. 250-265, 2007.

[16] H. Saeedi, N. Mollahasani, M. Mohseni Moghadam, and G. N. Chuev, "An operational Haar wavelet method for solving fractional Volterra integral equations," International Journal of Applied Mathematics and Computer Science, vol. 21, no. 3, pp. 535-547, 2011.

[17] A. H. Darani and M. Lakestani, "Numerical solution of integrodifferential equations usnig flatlet oblique multiwavelets," Dynamics of Continuous, Discrete and Impulsive Systems Series A, Mathematical Analysis, vol. 17, pp. 55-74, 2010.

[18] M. R. A. Darani, H. Adibi, R. P. Agarwal, and R. Saadati, "Flatlet oblique multiwavelet for solving integro-differential equations," Dynamics of Continuous, Discrete and Impulsive Systems Series A: Mathematical Analysis, vol. 15, no. 6, pp. 755-768, 2008.

[19] M. Lakestani, M. Dehghan, and S. Irandoust-Pakchin, "The construction of operational matrix of fractional derivatives using B-spline functions," Communications in Nonlinear Science and Numerical Simulation, vol. 17, no. 3, pp. 1149-1162, 2012.

[20] H. Saeedi, M. Mohseni Moghadam, N. Mollahasani, and G. N. Chuev, "A CAS wavelet method for solving nonlinear Fredholm integro-differential equations of fractional order," Communications in Nonlinear Science and Numerical Simulation, vol. 16, no. 3, pp. 1154-1163, 2011.

[21] I. Podlubny, Fractional Differential Equations, vol. 198 of Mathematics in Science and Engineering, Academic Press, San Diego, Calif, USA, 1999.

[22] L. Wang, Y. Ma, and Z. Meng, "Haar wavelet method for solving fractional partial differential equations numerically," Applied Mathematics and Computation, vol. 227, pp. 66-76, 2014.

[23] M. Yi and J. Huang, "Wavelet operational matrix method for solving fractional differential equations with variable coefficients," Applied Mathematics and Computation, vol. 230, pp. 383-394, 2014.

[24] K. Parand, A. R. Rezaei, and A. Taghavi, "Numerical approximations for population growth model by rational Chebyshev and Hermite functions collocation approach: a comparison," Mathematical Methods in the Applied Sciences, vol. 33, no. 17, pp. 2076-2086, 2010.

[25] A.-M. Wazwaz, A First Course in Integral Equations, World Scientific Publishing, Singapore, 1997.

[26] M. Yi and J. Huang, "Wavelet operational matrix method for solving fractional differential equations with variable coefficients," Applied Mathematics and Computation, vol. 230, pp. 383-394, 2014

[27] A.-M. Wazwaz, "Analytical approximations and padé approximants for volterra's population model," Applied Mathematics and Computation, vol. 100, no. 1, pp. 13-25, 1999.

[28] N. A. Khan, A. Mahmood, N. A. Khan, and A. Ara, "Analytical study of nonlinear fractional-order integrodifferential equation: revisit Volterra's population model," International Journal of Differential Equations, vol. 2012, Article ID 845945, 8 pages, 2012. 


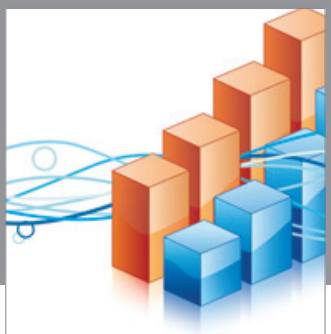

Advances in

Operations Research

vatem alat4

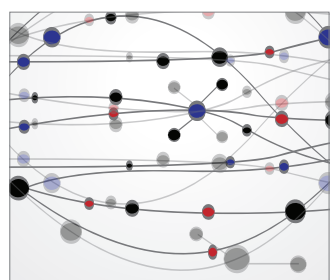

\section{The Scientific} World Journal
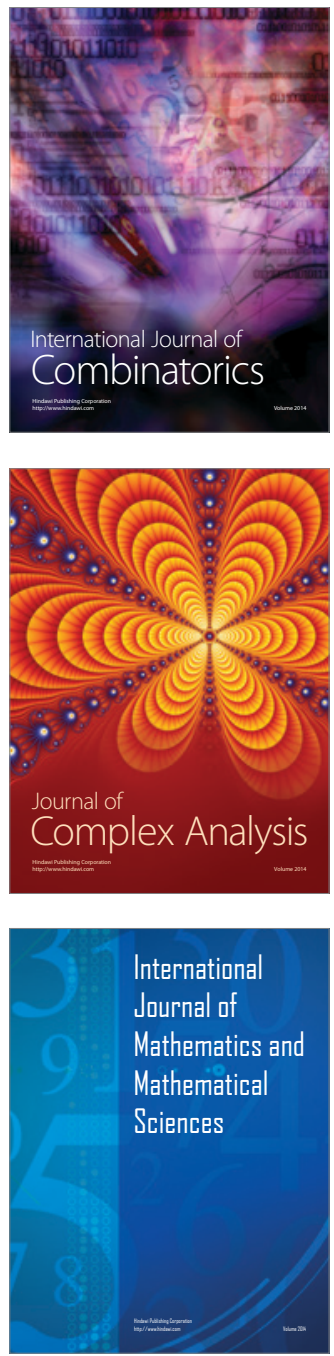
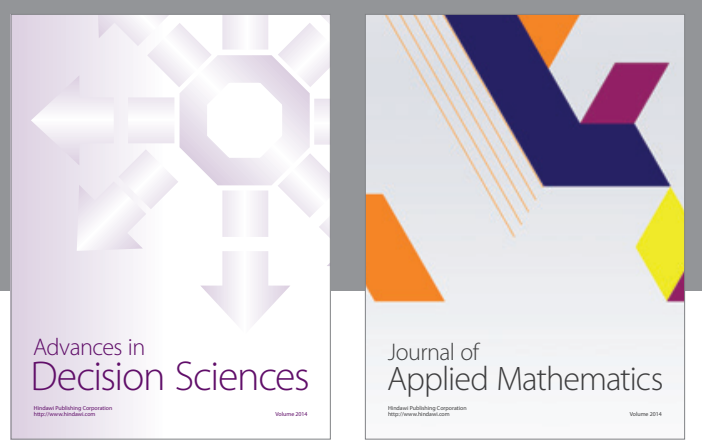

Algebra

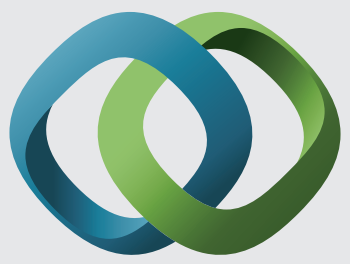

\section{Hindawi}

Submit your manuscripts at

https://www.hindawi.com
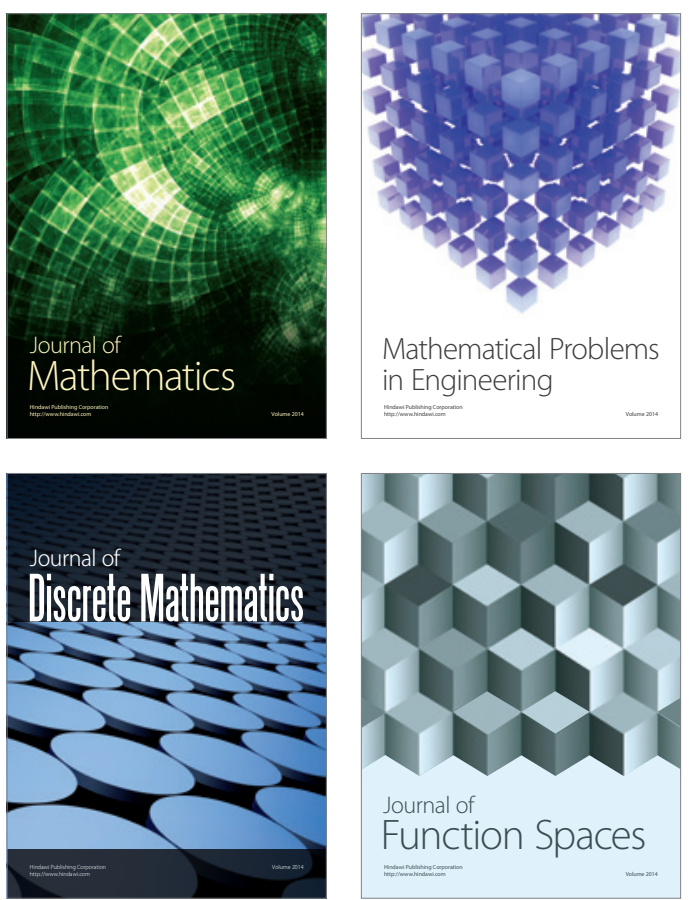

Mathematical Problems in Engineering
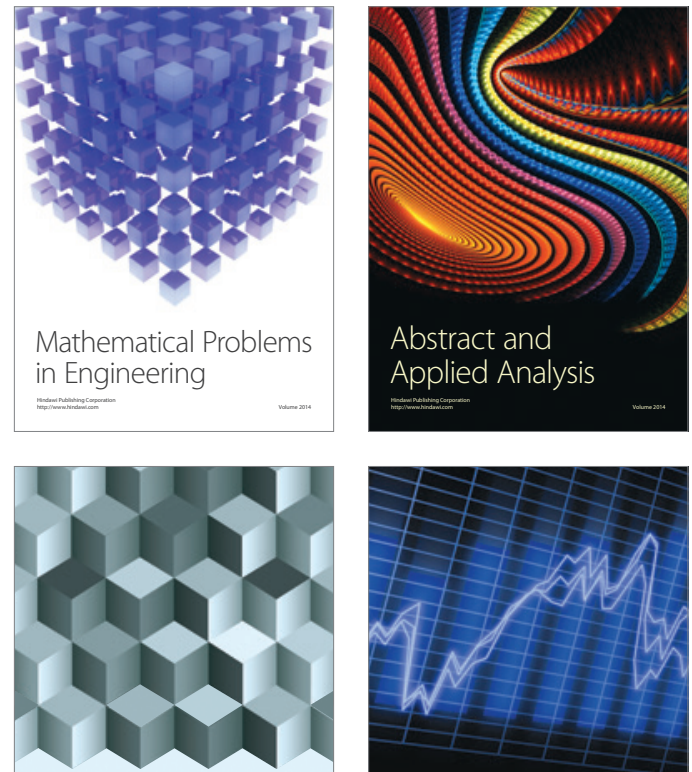

Journal of

Function Spaces

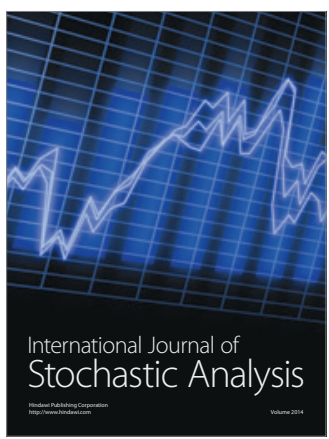

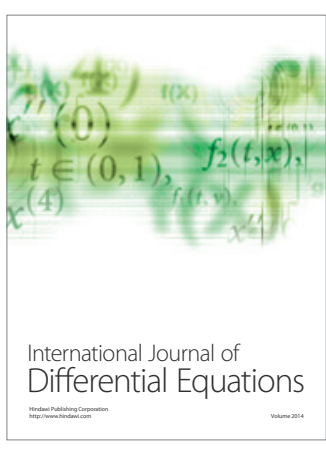
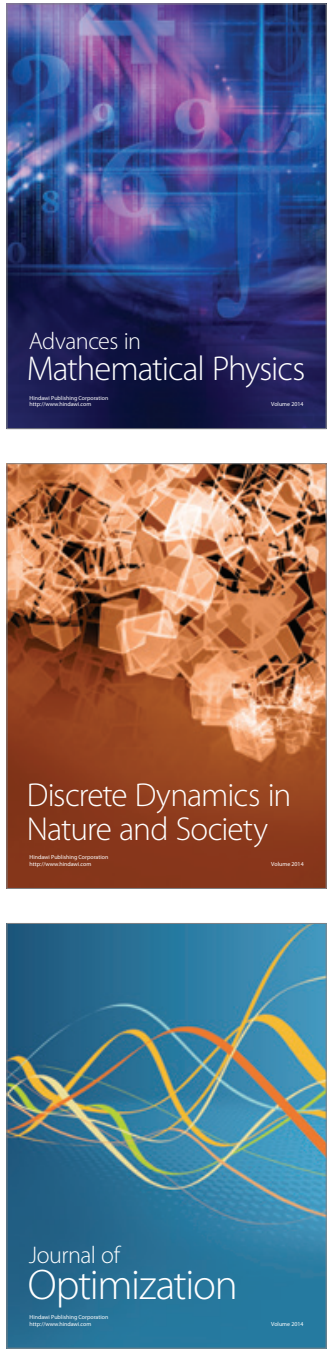